Pesq. Vet. Bras. 35(Supl.1):10-14, dezembro 2015

DOI: $10.1590 /$ S0100-736X2015001300003

\title{
Influência da altitude sobre parâmetros eletrocardiográficos, obtidos por meio do uso do "Holter" em cavalos de enduro em diferentes níveis de condicionamento físico ${ }^{1}$
}

\author{
Paulo R. Griska², Lílian E.S. Michima², Tiago M. Oliveira² e Wilson R. Fernandes* \\ ABSTRACT.- Griska P.R., Michima L.E.S., Oliveira T.M. \& Fernandes W.R. 2015. [Influence \\ of altitude on electrocardiographic parameters by means of the Holter system uti- \\ lization in endurance horses in different levels of physical conditioning.] Influência \\ da altitude sobre parâmetros eletrocardiográficos, obtidos por meio do uso do "Holter" \\ em cavalos de enduro em diferentes níveis de condicionamento físico. Pesquisa Veterinária \\ Brasileira 35(Supl.1):10-14. Departamento de Clínica Médica, Faculdade de Medicina Vete- \\ rinária e Zootecnia, Universidade de São Paulo, Av. Prof. Dr. Orlando Marques de Paiva 87, \\ São Paulo, SP 05508-270,, Brazil. E-mail: wilsonrf@usp.br \\ The aim of this paper was to study the influence of altitude on electrocardiographic \\ parameters by means of the Holter system in 40 Arabian and Arabian crossbred horses, at \\ different levels of physical conditioning, during training in trails of $15 \mathrm{~km}$ extension with \\ mean speed of $15 \mathrm{~km} / \mathrm{h}$, and altitude varying from sea level to $1700 \mathrm{~m}$ high. The electrocar- \\ diographic monitoring was obtained by standard electrocardiography at rest before exer- \\ cise, and by dynamic electrocardiography with the Holter system applied during exercise \\ and in post-exercise recovery. The animals presented sinusal rhythm, with a mean regis- \\ tered period for sinusal tachycardia of $32 \%$, and $14 \%$ for sinusal bradycardia. Among the \\ arrhythmias there was first-degree atrioventricular block (20\%) and second-degree atrio- \\ ventricular block (5\%). Supraventricular ectopic arrhythmias were observed in two horses \\ and only one showed ventricular arrhythmia. The number of asystoles was greater in less \\ conditioned horses. Mean and maximal heart rates were influenced by altitude, with higher \\ values in higher altitudes.
}

INDEX TERMS: Cardiology in horses, endurance riding, altitude, physical conditioning in horses, Holter system.

RESUMO.- 0 presente trabalho teve por objetivo o estudo da influência da altitude sobre os parâmetros eletrocardiográficos obtidos por meio do uso do Holter em 40 cavalos das raças Árabe e Cruza Árabe, em diferentes níveis de condicionamento físico, realizando treinamento em trilhas de $15 \mathrm{~km}$ de extensão com velocidade média de $15 \mathrm{~km} / \mathrm{h}$ e variando entre o nível do mar até $1700 \mathrm{~m}$ de altitude. 0 monitoramento eletrocardiográfico foi obtido por eletrocardiografia padrão em repouso antes do exercício e por eletrocardiografia dinâmica, com o uso do Holter, durante o

\footnotetext{
${ }^{1}$ Recebido em 2 de maio de 2015.

Aceito para publicação em 29 de novembro de 2015.

${ }^{2}$ Pós-Graduando do Departamento de Clínica Médica da Faculdade de Medicina Veterinária e Zootecnia (FMVZ), Universidade de São Paulo (USP), Av. Prof. Dr. Orlando Marques de Paiva 87, Cidade Universitária, São Paulo, SP 05508-27, Brasil.

${ }^{3}$ Docente do Departamento de Clínica Médica, FMVZ-USP, São Paulo, SP. *Autor para correspondência: wilsonrf@usp.br
}

exercício e na recuperação pós-exercício. Os animais apresentaram ritmo sinusal com período médio gravado para taquicardia sinusal (32\%) e bradicardia sinusal (14\%). Entre as arritmias observadas estão o bloqueio atrioventricular de primeiro grau (20\%) e o bloqueio atrioventricular de segundo grau (5\%). As arritmias ectópicas supraventriculares foram observadas em dois animais e somente um animal apresentou arritmia ventricular. 0 número de assistolias foi maior em animais menos condicionados. As frequências cardíacas médias e máximas sofreram efeito da altitude com valores maiores em altitude elevada.

TERMOS DE INDEXAÇÃO: Cardiologia em equinos, enduro equestre, altitude, condicionamento físico em equinos, sistema Holter.

\section{INTRODUÇÃO}

As provas de enduro equestre realizadas em distância de até $160 \mathrm{~km}$ e em diferentes condições ambientais relacio- 
nadas ao clima, tipo de terreno e altitude, impõe ao cavalo de enduro um esforço atlético significativo, exigindo do animal um alto nível de condicionamento físico.

Arritmias cardíacas são frequentes em cavalos e muitas vezes fisiológicas, sendo que estas acabam desaparecendo durante o exercício (Mitten 1996). A arritmia sinusal, bradicardia sinusal, bloqueio sinoatrial e parada sinoatrial associadas a tônus vagal tem sido achados comuns em cavalos sadios em repouso e normalmente desaparecem com o exercício (Reef 1999). Por esta característica intermitente, a eletrocardiografia contínua com o Holter tem sido usada na determinação dessas arritmias durante o exercício e avaliação do grau de comprometimento no desempenho atlético do animal.

Franceschi et al. (2000) propuseram uma técnica padrão para obtenção de gravação eletrocardiográfica pelo método Holter em cavalos trabalhando em esteira, e concluíram que a pequena dimensão do aparelho, a simplicidade na utilização e ausência de aparelhagem de suporte para gravação, torna o método de Holter apto para a gravação do eletrocardiograma durante o exercício, assim como Zucca et al. (2003) que usaram a eletrocardiografia contínua pelo sistema de Holter durante o exercício em esteira, monitorando os cavalos 15 minutos antes do exercício e até 30 minutos após o esforço, observando que a onda $\mathrm{P}$ é facilmente identificada e os complexos QRS apresentavam alta voltagem.

A morfologia da onda T após o esforço foi utilizada para estimar o estado de condicionamento atlético dos cavalos de corrida pelo método de Holter. Foi observada uma onda $\mathrm{T}$ positiva em DII em cavalos com bom condicionamento físico e onda T negativa ou difásica em cavalos não condicionados, nos dez minutos após o esforço (Baha 1991).

A hipótese de que a altitude tem efeitos significativos sobre os parâmetros fisiológicos em cavalos competindo em eventos de três dias e em provas preliminares no Colorado (1900m acima do nível do mar) comparando com provas similares no Arizona (ao nível do mar) foi testada por Foreman et al. 1999, que observaram no pós-exercício, no Colorado, aumento da frequência cardíaca, frequência respiratória, diminuição nos valores de cálcio e potássio quando comparada com os valores ao nível do mar. Wickler \& Anderson (2000) não constataram diferenças estatisticamente significativas no pico de frequência cardíaca e lactato em teste padronizado em esteira pré-altitude e na pós-altitude concluindo que não houve efeito da aclimatação na altitude, mas observaram diminuição no tempo de recuperação para ambas as variáveis, sugerindo um efeito positivo da aclimatação na altitude.

Em virtude dos poucos conhecimentos do uso do método de Holter em medicina veterinária, realizamos este trabalho com a finalidade de traçarmos os parâmetros eletrocardiográficos com monitoração contínua durante o exercício em trilha e pós-exercício em cavalos de enduro, em diferentes altitudes e condicionamentos físicos.

\section{MATERIAL E MÉTODOS}

Foram utilizados 40 animais da espécie equina, machos ou fêmeas, das raças Puro Sangue Árabe, Anglo Árabe e Mestiços de Árabe
(Cruza Árabe), com idade variando de 5 a 14 anos, clinicamente sadios, realizando treinamentos em trilhas de $15 \mathrm{~km}$ de distância, com velocidade média de $15 \mathrm{~km}$ por hora, três vezes por semana em diferentes regiões do Estado de São Paulo. Em relação ao condicionamento físico, os animais foram divididos em três categorias: C1, animais em início de treinamento $(n=13)$; $C 2$, animais em treinamento e competição em provas de até $80 \mathrm{~km}$ de distância $(n=13) ; C 3$, animais em treinamento e competição em provas acima de $80 \mathrm{~km}$ de distância (n=14).

Os animais foram avaliados por meio de exames clínicos, laboratoriais, eletrocardiografia padrão e monitoração eletrocardiográfica contínua pelo sistema Holter. Foram exercitados em diferentes regiões do Estado de São Paulo com condições de altitude que variavam do nível do mar até $1700 \mathrm{~m}$, assim como diferentes condições de temperatura e umidade que eram $14-39^{\circ} \mathrm{C}$ e $19-78 \%$, respectivamente, monitoradas por higrômetro, marca ALLA ${ }^{\circledR}$, modelo 7429. Os locais onde se realizaram as colheitas dos dados foram divididos com relação à altitude em alto $(1700 \mathrm{~m})$, médio (900m) e baixo (nível do mar), para efeito de análise estatística. Os três grupos foram avaliados nas três diferentes altitudes.

A eletrocardiografia padrão foi realizada em repouso antes do exercício, utilizando eletrocardiógrafo da marca TEB ${ }^{\circledR}$, modelo ECGPC, acoplado a notebook COMPAQ ${ }^{\circledR}$, modelo ARMADA 1750, com software padrão instalado para avaliação dos traçados. Os traçados eletrocardiográficos foram realizados segundo o método preconizado por Fregin (1982).

A gravação eletrocardiográfica contínua pelo sistema Holter, durante e após exercício, foi realizada pelo uso do gravador de Holter, marca Dýnamis (Cardios), modelo 4000, de três derivações, com cabo do paciente de quatro vias, que utiliza fita magnética tipo cassete comum (BASF ${ }^{\circledR}$ ferro) e duas pilhas comuns para seu funcionamento. 0 gravador foi acondicionado em uma bolsa e fixado no lado esquerdo da sela, próximo a cernelha. Em relação ao cabo do paciente, o branco acumula as funções de negativo comum dos três canais e o terra; o cabo vermelho, o laranja, e o preto são, respectivamente, os eletrodos positivos dos canais 1, 2 e 3 (MV1-2, MV3 e MV5).

Foram usados eletrodos descartáveis feitos para humanos, marca MADTRACE ${ }^{\circledR}$, por terem melhor condutibilidade e serem mais estáveis (Scheffer et al. 1996). Os locais de fixação dos eletrodos foram limpos e receberam uma dose extra de cola nas bordas do limite pré-demarcado (Super Bonder ${ }^{\circledR}$, Loctite Brasil). Não foi realizada tricotomia porque a fixação da cola sobre os pelos mostrou-se mais efetiva. Os eletrodos negativo e neutro (cabo branco) foram colocados na proeminência do manúbrio; o positivo do canal 1 (cabo vermelho) foi colocado a esquerda da linha média ventral, próximo a cartilagem xifóide, aproximadamente $10 \mathrm{~cm}$ atrás da barrigueira; o positivo do canal 2 (cabo amarelo) foi colocado a esquerda da linha média ventral, $10 \mathrm{~cm}$ acima da altura do olecrano e $10 \mathrm{~cm}$ atrás da barrigueira; e o positivo do canal 3 (cabo preto) foi colocado no lado esquerdo da cernelha, a frente do ângulo da escápula. Foram adotadas posições próximas aquelas referendadas por Scheffer et al. (1996), para possibilitar a colocação da sela e assim evitar qualquer interferência da perna do cavaleiro.

0 aparelho era ligado e o horário ajustado (tempo zero) antes do início do exercício, permanecendo ligado até o animal atingir, na sua recuperação, 20\% acima da frequência cardíaca basal, momento no qual o aparelho era desligado.

Os resultados obtidos foram avaliados por meio de análise de variância para avaliação dos efeitos da altitude, do condicionamento físico e dos efeitos de interação altitude/condicionamento, por programa de computador STATISTICA versão 6.0. Foram realizadas comparações pareadas das médias utilizando o teste de Tukey $(\mathrm{p}=5 \%)$. 
Quadro 1. Índice da Morfologia da onda T, segundo Baha (1991)

\begin{tabular}{lc}
\hline \multicolumn{1}{c}{ Morfologia da onda $\mathrm{T}$} & Índice \\
\hline Monofásica $+\operatorname{com}|\mathrm{T}| \approx|\mathrm{S}|$ & 6 \\
Monofásica $+\operatorname{com}|\mathrm{T}| \leq 1 / 2|\mathrm{~S}|$ & 4 \\
Difásica com predominância + & 2 \\
Difásica com componentes = & 0 \\
Difásica com predominância - & -2 \\
Monofásica $-\operatorname{com}|\mathrm{T}| \leq 1 / 2|\mathrm{~S}|$ & -4 \\
Monofásica $-\operatorname{com}|\mathrm{T}| \approx|\mathrm{S}|$ & -6
\end{tabular}

Em relação à avaliação da morfologia da onda T, foi utilizado o mesmo critério de índice de morfologia de onda T sugerido por Baha (1991) mostrado no Quadro 1. A análise estatística foi realizada nos dois canais 1 e 2 e em três momentos: repouso, final do esforço e em 10 minutos de recuperação.

\section{RESULTADOS}

As gravações pelo sistema Holter foram realizadas em boas condições técnicas em ambos os canais 1 e 2 , tanto durante o exercício como no período de recuperação. Os traçados eletrocardiográficos produzidos pelo Holter nas duas derivações foram idênticos, sendo caracterizados no início do exercício por uma onda P bífida e positiva, complexo QRS com característica RS, com amplitude maior no canal 1, e onda T variando de monofásica positiva a difásica com predominância positiva. Durante o esforço, no período de frequência cardíaca máxima, a onda P aumentava a amplitude e perdia a característica bífida, o segmento ST desaparecia e a onda T tornava-se muito positiva e com grande amplitude. No final do esforço, a onda $\mathrm{P}$ voltava à característica bífida, os intervalos voltavam a ser identificados e a onda $\mathrm{T}$ variava de monofásica positiva a difásica, com predominância positiva.

Os animais apresentavam ritmo sinusal sendo que o percentual médio do período gravado foi de $32 \%$ para taquicardia sinusal e de $14 \%$ para bradicardia sinusal. Durante a gravação, oito animais (20\%) apresentaram bloqueio atrioventricular de primeiro grau, dois (5\%) bloqueio atrioventricular de segundo grau e nenhum animal apresentou episódios de depressão ou elevação do segmento ST.

Em relação às arritmias ectópicas ventriculares, um único animal apresentou este tipo de ectopia e de forma isolada, sendo que a relação entre o número total de complexos QRS e o número de arritmias ectópicas ventriculares foi menor que $1 \%$. As arritmias ectópicas supraventriculares foram observadas em dois animais, de forma isolada, sendo que um apresentou um episódio e o outro cinco episódios. A relação entre o número total de complexos QRS e o número de arritmias supraventriculares foi menor que $1 \%$ em ambos os animais.

Os resultados da análise de variância mostram que a altitude teve efeito significativo $(p=5 \%)$ sobre a frequência cardíaca média e máxima na altitude alta quando comparado com as outras altitudes (Quadro 2). As médias da frequência cardíaca mínima segundo as altitudes baixa, média e alta não diferiram significativamente entre si.

Em relação ao número de assistolias (pausas maiores que dois segundos), foram observadas em onze animais $(27,5 \%)$, sendo que um animal apresentou pausa de 5 se-
Quadro 2. Médias desvios padrões para as médias da freqüência cardíaca mínima, média e máxima segundo altitude, obtidas por meio do Holter em cavalos de enduro em treinamento

\begin{tabular}{cccc}
\hline Altitude & FCmin & FCmed & Fcmáx \\
\hline Baixa & $35.9 \pm 2,69 \mathrm{a}$ & $85,7 \pm 2.69 \mathrm{~b}$ & $144,9 \pm 6.01 \mathrm{~b}$ \\
Média & $33.7 \pm 2,16 \mathrm{a}$ & $86,0 \pm 2.16 \mathrm{~b}$ & $153,8 \pm 4.83 \mathrm{~b}$ \\
Alta & $37.9 \pm 2,26 \mathrm{a}$ & $97,8 \pm 2.26^{\mathrm{a}}$ & $175,3 \pm 3.06 \mathrm{a}$
\end{tabular}

Médias com letras em comum numa mesma coluna, indicam amostras que não diferem significativamente entre si $(\mathrm{p}=5 \%)$.

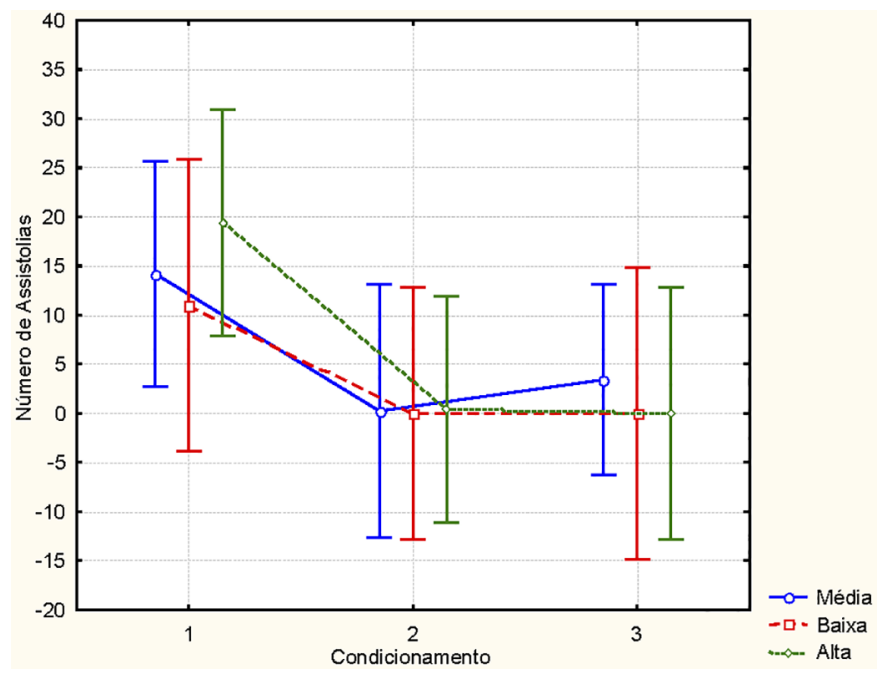

Fig.1. Efeitos da altitude e condicionamento físico sobre o número de assistolias, obtida na eletrocardiografia dinâmica "Holter", realizada em cavalos de enduro em treinamento. São Paulo, 2003.

Quadro 3. Médias e desvios padrões para as médias do número de assistolias e maior pausa (segundos), segundo condicionamento físico, obtidas por meio do Holter, em cavalos de enduro em treinamento

\begin{tabular}{ccc}
\hline Condicionamento & $\begin{array}{c}\text { Média (1) } \\
\text { № de assistolias }\end{array}$ & $\begin{array}{c}\text { Média (1) } \\
\text { Maior Pausa (segundos) }\end{array}$ \\
\hline 1 & $14.9 \pm 3.60 \mathrm{a}$ & $1.68 \pm 0.40^{\mathrm{a}}$ \\
2 & $0.2 \pm 3.51 \mathrm{~b}$ & $0.49 \pm 0.39 \mathrm{~b}$ \\
3 & $1.1 \pm 3.58 \mathrm{~b}$ & $0.21 \pm 0.40 \mathrm{~b}$
\end{tabular}

(1)médias com letras em comum numa mesma coluna, indicam amostras que não diferem significativamente entre $\mathrm{si}(\mathrm{p}=5 \%)$.

gundos. Nas médias e desvios padrões do número de assistolias e maior pausa (segundos) entre as altitudes baixa, média e alta, não foram observados efeito significativo entre as altitudes (Fig.1). 0 Quadro 3 mostra que a média do número de assistolias e maior pausa (segundos) para o condicionamento físico 1 foi significativamente maior do que as médias do condicionamento físico 2 e 3 ( $p=5 \%)$.

$\mathrm{Na}$ avaliação da morfologia da onda $\mathrm{T}$ foi observado que houve efeito significativo desses momentos e da altitude nos resultados da análise de variância, enquanto o condicionamento físico não teve efeito significativo em ambos os canais (canal 1 e 2). Nos Quadros 4 e 5 são apresentadas as médias e desvios padrões para as médias do índice de onda $\mathrm{T}$ (canal 1 e 2) segundo momento e altitude, respectivamente. Pelo teste de Tukey, a média do índice de onda T no 
Quadro 4. Médias e desvios padrões para as médias do índice de onda $\mathrm{T}$ (canal 1 e 2) segundo momento, obtidas por meio do Holter , em cavalos de enduro em treinamento

\begin{tabular}{lcc}
\hline \multicolumn{1}{c}{ Momento } & Média* $^{*}$ & Média* $^{*}$ \\
& Índice onda T (canal 1) & Índice onda T (canal 2) \\
\hline Repouso & $2.4 \pm 0.43 \mathrm{~b}$ & $2.9 \pm 0.45 \mathrm{~b}$ \\
Final do Esforço & $4.4 \pm 0.51 \mathrm{a}$ & $4.6 \pm 0.50^{\mathrm{a}}$ \\
Recuperação & $3.7 \pm 0.48^{\mathrm{a}}$ & $3.2 \pm 0.50 \mathrm{~b}$
\end{tabular}

* Médias com letras em comum numa mesma coluna, indicam amostras que não diferem significativamente entre si $(\mathrm{p}=5 \%)$.

Quadro 5. Médias e desvios padrões para as médias do índice de onda $\mathrm{T}$ (canal 1 e 2) segundo altitude, obtidas por meio do Holter, em cavalos de enduro em treinamento

\begin{tabular}{|c|c|c|}
\hline Altitude & $\begin{array}{c}\text { Média* } \\
\text { Índice onda T (canal 1) }\end{array}$ & $\begin{array}{c}\text { Média* } \\
\text { Índice onda T (canal 2) }\end{array}$ \\
\hline Média & $4.6 \pm 0.41^{\mathrm{a}}$ & $3.0 \pm 0.54 \mathrm{a}$ \\
\hline Baixa & $2.5 \pm 0.54 b$ & $4.4 \pm 0.43 a$ \\
\hline Alta & $3.4 \pm 0.46 \mathrm{~b}$ & $3.2 \pm 0.47 \mathrm{a}$ \\
\hline
\end{tabular}

* Médias com letras em comum numa mesma coluna, indicam amostras que não diferem significativamente entre si $(\mathrm{p}=5 \%)$.

final de esforço e na recuperação foram significativamente maiores em relação a média do índice em repouso $(\mathrm{p}=5 \%)$ e, em relação a altitude no canal 1 , a média do índice na altitude média foi significativamente maior às médias nas altitudes baixa e alta $(\mathrm{p}=5 \%)$, diferentemente dos resultados obtidos no canal 2.

\section{DISCUSSÃO}

Os resultados do presente trabalho mostraram uma satisfatória qualidade de gravação do eletrocardiograma em ambos os canais pelo sistema Holter, durante o exercício e com baixa quantidade de artefatos, semelhante aos resultados observados por Raekallio (1992), Scheffer et al. (1995, 1996), Franceschi et al. (2000) e Zucca et al. (2003). O posicionamento, o tipo e a fixação dos eletrodos são descritos como importantes fatores para obtenção de um sinal livre de artefatos (Raekallio 1992, Scheffer et al. 1996). Corroborando com as observações feitas por Scheffer et al. (1996), este trabalho permitiu afirmar que os eletrodos descartáveis feitos para utilização em humanos, com uma dose extra de cola e sem a realização da tricotomia no animal, mostraram-se eficazes quanto à fixação e condutibilidade do sinal.

As posições adotadas para os eletrodos evitaram interferências tanto da sela quanto da perna do cavaleiro, durante a gravação com o animal executando o exercício. A desvantagem na fixação dos eletrodos com dose extra de cola é a dificuldade na remoção dos eletrodos, o que criou áreas de alopecia nos animais nos pontos de fixação, fato este também descrito por Scheffer et al. (1995).

As características do traçado eletrocardiográfico em ambos os canais gravados pelo sistema Holter assemelham-se às observações descritas por Scheffer et al. (1996) em equinos analisados em teste padrão em esteira, porém foram discordantes em relação à voltagem e estabilidade do sinal no canal 2 durante o exercício, sendo que no presente estudo observou-se estabilidade em ambos os canais e maior voltagem no canal 2, fato este resultante do posicionamento dos eletrodos que não sofreram movimentação significativa.

Dois animais (5\%) apresentaram bloqueio atrioventricular de segundo grau neste trabalho, sendo que um deles apresentou a mesma arritmia em repouso em eletrocardiografia padrão, mas em ambos os animais o bloqueio foi observado somente no início do exercício (ao passo) com baixa frequência cardíaca, semelhante aos resultados observados por Raekallio (1992), Scheffer et al. (1995) e Kuwahara et al. (1999), sendo esta condição resultante do aumento do tônus vagal.

No presente estudo dois animais (5\%) em bom condicionamento físico apresentaram arritmias ectópicas supraventriculares, sendo um durante o exercício e outro ao final do esforço, resultado semelhante aos achados por Raekallio (1992) e Scheffer et al. (1996) com animais sadios, sendo que o resultado neste trabalho provavelmente ocorreu por aumento da atividade simpática.

Em relação às arritmias ectópicas ventriculares, os resultados observados nesse estudo mostraram que um animal $(2,5 \%)$ apresentou e de forma isolada este tipo de arritmia, semelhante aos resultados obtidos por Scheffer (1996) e Kuwahara (1999), mas inferior às observações de Bonagura \& Reef (2000) que descreveram a incidência de $14 \%$ de complexos ventriculares prematuros, durante monitoração eletrocardiográfica contínua de 24 horas.

Os valores médios da frequência cardíaca mínima em diferentes condicionamentos físicos variaram de 32,8 $\pm 2,41$ a 40,1 $\pm 2,35$ bpm obtidas no início e ao final do exercício com o animal ao passo e encilhado, sendo diferentes dos resultados obtidos por Scheffer $(1995,1996)$ nos quais os animais tiveram médias $79 \pm 8$ a $84 \pm 7 \mathrm{bpm}$ em teste padronizado em esteira, sendo que a diferença nos resultados pode ser atribuída ao estresse de adaptação a esteira. Em relação às médias das frequências cardíacas média e máxima, o estudo mostrou o efeito da altitude alta com aumento significativo desses valores com em comparação com as outras altitudes, resultado semelhante ao observado por Foreman et al. (1999).

No presente trabalho, foram observadas assistolias (pausas maiores que dois segundos) em onze animais $(27,5 \%)$ sendo que a maior pausa foi de 5 segundos; o nível de condicionamento físico $\mathrm{C} 1$ teve um efeito significativo sobre o número de assistolias e assim como o tempo de maior pausa em segundos, já que nesse condicionamento a média do número de assistolias foi de $14,9 \pm 3$,6 e a média da maior pausa foi de $1,68 \pm 0,40$ segundos, resultado inferior ao observado por Viitasalo et al. (1982) em atletas humanos de endurance nos quais $37,1 \%$ apresentaram assistolias acima de 2 segundos.

$\mathrm{Na}$ avaliação da morfologia da onda T foi observado que houve efeito significativo desses momentos e da altitude nos resultados da análise de variância, enquanto o condicionamento físico não teve efeito significativo em ambos os canais. No presente estudo os animais apresentaram no repouso índice superior a 2,0, tanto no canal 1 como no canal 2 , caracterizando uma onda T difásica, com predominância positiva em ambos os canais; no final do esforço, os índices 
foram superiores a 4,0 em ambos os canais, caracterizando uma onda T monofásica positiva; e na recuperação os índices foram superiores a 3,0 em ambos os canais, caracterizando uma onda T difásica positiva tendendo a monofásica positiva.

Os resultados em relação à análise do momento de avaliação da onda T mostraram índices menores que os observados por Baha (1991), para cada momento em ambos os canais, mas demonstrando uma tendência de característica de morfologia da onda T semelhante para cavalos avaliados em boa forma física. Essa diferença pode estar relacionada à intensidade do esforço imposta aos animais por um exercício submáximo de longa duração quando comparado ao exercício intenso e de curta duração. A morfologia da onda T mostrou um efeito significativo da altitude média indicando uma tendência de maior potencial de condicionamento físico, pois a média do condicionamento nessa altitude foi ligeiramente maior que nas outras altitudes.

Os resultados referentes ao tempo de recuperação da frequência cardíaca após o exercício não tiveram efeito significativo tanto na altitude, no condicionamento físico e na interação de ambos, mostrando somente uma tendência de serem menores na baixa altitude e nos animais mais bem condicionados, resultados semelhantes aos de Skarda et al. (1976) em estudo dos efeitos do treinamento sobre a frequência cardíaca, no qual observaram que embora os valores médios diminuíssem com o treinamento no repouso e pós-exercício, os achados não apresentaram alterações significativas para avaliação do condicionamento físico.

\section{CONCLUSÕES}

A análise dos resultados obtidos no presente trabalho permitiu concluir que a metodologia utilizada para monitoração eletrocardiográfica contínua com o uso do Holter proporciona uma avaliação eletrocardiográfica fidedigna durante o exercício em trilha nos cavalos de enduro; as pausas são frequentes nestes animais e apresentam relação negativa com condicionamento físico; e a altitude aumento da frequência cardíaca média e máxima na altitude alta a alterações na morfologia da onda $\mathrm{T}$ em ambos os canais na altitude média.

Os resultados obtidos neste trabalho indicam que o grau de condicionamento e a altitude do local onde os animais são treinados tem influência nas variáveis cardíacas analisadas e merecem mais estudos para que se estabeleça uma relação entre estes resultados e a performance dos equinos que competem em provas de enduro.

\section{REFERÊNCIAS}

Baha S. 1991. Estimation de l'état de forme des chevaux de course par la morphologie de l'onde $\mathrm{T}$ après effort. Étude par la méthode de Holter. Revue Méd. Vét. 142(2):125-129.

Bonagura J.D. \& Reef V.B. 2000. Doenças cardiovasculares, p.250-319. In: Reed S.M. \& Bayly W.M. (Eds), Medicina Interna Equina. Editora Guanabara Koogan, Rio de Janeiro.

Foreman J.H., Waldsmith J.K. \& Lalum R.B. 1999. Environmental stress and 3-day eventing: effects of altitude. Equine Vet. J. 30(Suppl.):394-397.

Franceschi A., Bizzeti M., Sgorbini M. \& Casini L. 2000. Holter recording in equine cardiology: Exposing a standard technique. Annali della Facoltà di Medicina Veterinaria di Pisa 53:167-176.

Fregin, G.F. 1982. The equine electrocardiogram with stand-ardized body and limb positions. Cornell Vet. 72:304-324.

Kuwahara M., Hiraga A., Kai M., Tsubone H. \& Sugano S. 1999. Influence of training on autonomic nervous function in horses: evaluation by power spectral analysis of heart rate variability. Equine Vet. J. 30(Suppl.): 178-180.

Mitten L.A. 1996. Cardiovascular causes of exercise intolerance. Vet. Clin. North Am., Equine Pract. 12(3):473-493.

Raekallio M. 1992. Long term ECG recording with Holter monitoring in clinically healthy horses. Acta Vet. Scand. 33(1):71-75.

Reef V.B. 1999. Arrhythmias, p.180-196. In: Marr C.M. (Ed.), Cardiology of the Horse. W.B. Saunders, Philadelphia.

Scheffer C.W.J., Robben J.H. \& Sloet van Oldruitenborgh-Oosterban M.M. 1995. Continuous monitoring of ECG in horses at rest and during exercise. Vet. Rec.137:371-374.

Scheffer C.W.J. \& Sloet van Oldruitenborgh-Oosterban M.M. 1996. Computadorized ECG recording in horses during a standardized exercise test. Vet. Rec. 18:2-7.

Skarda R., Muir W.W., Milne D.W. \& Gabel A.A. 1976. Effects of training on resting and postexercise ECG in Standarbred horses, using a standardized exercise test. Am. J. Vet. Res. 37:1485-1488.

Viitasalo M.T., Kala R. \& Eisalo A. 1982. Ambulatory eletrocardiographic recording in endurance athletes. Brit. Heart J. 47:213-220.

Wickler S.J. \& Anderson T.P. 2000. Haematological changes and athletic performance in horses in response to high altitude $(3,800 \mathrm{~m})$. Am. J. Physiol., Regulatory Integrative Compendium Physiology 279:11761181.

Zucca E., Ferruci F., Di Fabio V., Croci C. \& Ferro E. 2003. The use of electrocardiographic recording with holter monitoring during treadmill exercise cardiac arrhythmias in racehorse. Vet. Res, Commun. 27(Suppl.1): 811-814. 